CI:1.25-2.36), and did not use condoms with last sex (OR 1.50, 95\% CI:1.05-2.16).

Conclusion Findings indicate that lottery-based incentives might be a feasible approach to increase syphilis testing uptake in Chinese MSM, particularly amongst men with higher risk attitude scores and sexually risky behaviors.

Disclosure No significant relationships.

\section{P743 CLINICAL PROFILES OF 58 HOSPITALIZED HIV-NEGATIVE PATIENTS WITH NEUROSYPHILIS IN A GENERAL HOSPITAL IN CHINA}

${ }^{1}$ Minzhi Wu*, ${ }^{2}$ Jun Dai, ${ }^{2}$ Jingjing Li, ${ }^{2}$ Xingfan Mo, ${ }^{2}$ Mingzhi Feng, ${ }^{2}$ Yating $\mathrm{Xu}$ ${ }^{3}$ Qianqiu Wang. 'The Fifth People's Hospital of Suzhou, Suzhou, China; ${ }^{2}$ The Fifth People's Hospital of Suzhou, Suzhou, China; ${ }^{3}$ Institute of Dermatology, Chinese Academy of Medical Sciences and Peking Union Medical College, Nanjing, China

\subsection{6/sextrans-2019-sti.803}

Background Patients with neurospyphilis have been increasingly reported from clinics in China. Symptomatic neurosyphilis is more common among HIV-positive than HIV-negative patients. Clinical data of neurosyphilis among HIV-negative patients are limited.

Methods Socio-demogeaphic and clinical data of the patients diagnosed with neurosyphilis and hospitalized at the Suzhou $5^{\text {th }}$ People's Hospital in China during January 2012 to November 2018 were collected and clinical and laboratory characteristics of these patients were analysed.

Results Of the 58 patients enrolled into the analysis, majority $(84.5 \%)$ were males. Two cases $(3.4 \%)$ were diagnosed with asymptomatic neurosyphilis by evidence of only increases of protein and white blood cell count in their cerebrospinal fluids (CSF). The clinical charactistics were presented to be meningeal vascular type (defined as presentation of hemiplegia, headache, tinnitus, or epileptic attack) among 6, paralytic dementia (defined as decline in intelligence and memory, decreas of judgement sense and cognition, or mental symptoms) among 30, tabes dorsalis (defined as having manifestation such as walking instability of lower limbs, lightning pain, numbness, abnormal urination, or Arrow pupil) among 8, and ocular syphilis (defined as choroiditis,iritis, retinitis, or optic atrophy) among 12 patients, respectively. Most of the patients $(84.5 \%)$ had serum RPR titers of $\geq 1: 8$ and two-thirds (75.9\%) were positive for RPR in CSF (ranging 1:1 to 1:16). Additional CSF evaluations indicated an elevated protein in 55 and leukopenia in 57 patients. Among 30 patients with paralytic dementia, 16 (53.3\%) shown a multiple lacunar foci in their brain CT or MRI, and 7 (23.3\%) had cerebral atrophy. Conclusion Symptomatic neurosyphilis is common among HIVnegative patients and clinical features charactierislized majorly as neurological, psychiatric or phthalmic symptoms have call for attendtion of the relevant departments to detect these patients for interventions timely.

Disclosure No significant relationships.

\section{P745 THE PREGNANCY SYPHILIS CASCADE OF CARE: "95-95- 95" GOALS FOR REDUCING OF CONGENITAL SYPHILIS IN THE STATE OF SAO PAULO, BRAZIL}

Carmen Silvia Domingues*, Mariza Tancredi, Valdir Pinto, Carla Luppi, Solange Gomes, Rosa Alencar, Maria Clara Gianna, Artur Kalichman. STI/AIDS Reference Center - Sao Paulo State Program of STI/AIDS, Sao Paulo Department of Health, Sao Paulo, Brazil

\subsection{6/sextrans-2019-sti.804}

Background Congenital syphilis (CS) is a preventable condition. However, in 2017, 4,039 cases were reported in São Paulo State (SPS), with an incidence rate of 6.6 cases $/ 1,000$ live births (LB). In 2018, SPS proposed the "95-95-95" goals to reduce CS: $95 \%$ of all pregnant women (PW) with timely diagnosis during antenatal care (ANC), 95\% of all with timely diagnosis receiving treatment and $95 \%$ of all babies from treated PW-syphilis, born without CS. This study aimed evaluates the pregnancy syphilis cascade of care and the "95-9595" goals for reducing CS in SPS, 2017.

Methods Ecological study, using reported cases of PW-syphilis and CS. The cascade of PW-syphilis care was estimated considering five columns. First column: number of PW-syphilis, calculated considering an estimated prevalence of $2.1 \%$ of syphilis in PW; and for the total population of PW, was considered the number of LB increased by $10 \%$ for fetal losses. Second: PW-syphilis diagnosed during ANC or delivery. Third: PW-syphilis linked to ANC with timely diagnosis. Fourth: PWsyphilis properly treated. Fifth: potentially avoided CS cases.

Results Were estimated 14,369(100\%) PW-syphilis, of these, $82.6 \%(\mathrm{~N}=11,875)$ were diagnosed during ANC or delivery; $73.8 \%(\mathrm{~N}=10,606)$ linked to ANC with timely diagnosis; $66.1 \%(\mathrm{~N}=9,500)$ treated and $54.5 \%(\mathrm{~N}=7,836)$ CS cases avoided. For the "95-95-95" goals, SPS reached the following levels: $89.3 \%(\mathrm{~N}=10,606 / 11,875)$ of PW-syphilis with diagnosis during $\mathrm{ANC} ; 89.6 \%(\mathrm{~N}=9,500 / 10,606)$ were timely treated and $82.5 \%(\mathrm{~N}=7,836 / 9,500)$ of babies born without CS.

Conclusion Prevention actions should be intensified to improve access and qualification of ANC. Specifically: wide screening coverage, availability of medication for the timely treatment and reproductive planning. These challenges can be overcome with an agenda of priorities and political commitment. The integration of STI/HIV/AIDS and maternal-child health programs, as well as the strengthening of primary care, is essential for the sustainability, duration and success of interventions aimed to the CS elimination.

Disclosure No significant relationships. 